\title{
Gastrointestinal Parasitosis in the Population of the City of N'Djamena [Chad]: Causes of Their Persistence
}

\author{
Hamit Mahamat Alio (Corresponding author)
}

Faculty of Human Health Sciences, University of N ${ }^{e e}$ Djamena, P.O. Box 1117, Nee Djamena, Chad, Faculty of Sciences, University of Yaoundé 1, P.O. Box 812 Yaoundé, Cameroon. E-mail: hamitalio@yahoo.fr

\section{Fombotioh Ndifor}

University of N'Djamena, Faculty of Human Health Sciences, PO Box 1117, N'Djamena, Chad, Cameroon. E-mail: fombotioh2020@gmail.com

\section{Samafou Kemba}

Laboratory of Parasitology and Ecology, Faculty of Sciences, PO Box 812 Yaoundé, Cameroon. E-mail: kembasamafou@yahoo.fr

Issa Ramat Adam

University of Douala, Faculty of Sciences, PO Box 24157 Douala, Cameroon, University of N'Djamena, Faculty of Human Health Sciences, PO Box 1117, N'Djamena, Chad, Cameroon.

E-mail: issaramat2@gmail.com

\section{Nack Jacques}

University of Douala, Faculty of Sciences, PO Box 24157 Douala, Cameroon.

E-mail: jacquesnack@yahoo.fr

\section{Bilong Bilong Charles Felix}

Faculty of Sciences, University of Yaoundé 1, P.O. Box 812 Yaoundé, Cameroon.

E-mail: bilong_bilong@yahoo.com

Received: October 9, 2020 Accepted: October 26, 2020

doi:10.5296/jbls.v11i2.17891 URL: https://doi.org/10.5296/jbls.v11i2.17891 


\section{Abstract}

In tropical areas gastrointestinal parasitosis are constantly changing in frequency and the large number of asymptomatic carriers continue to be a public health problem. This study was carried out during the last trimester of 2019 in the city of N'Djamena (Chad). This work was designed to take a stock of the overall level of carriage of parasitic infections of the population of the city. Our study sample was made up of 366 individuals whose age varied from 1 to 77 years. Each subject included in this study benefited from parasitological stool analysis using three methods. The method of direct observation in physiological water, the method of concentration in formalin-ether and that of Kato Katz. The results obtained showed that 222 subjects were carriers of at least one species of parasite, or either a global infection rate of $60.66 \%$. Ten species of gastrointestinal parasites were identified of which three species of protozoa: Entamoeba histolytica/dispar (34.70\%), Giardia intestinalis (3.55\%), Entamoeba coli $(0.55 \%)$ and seven species of helminths: Hymenolepis nana (18.85\%), Ascaris lumbricoides (9.29\%), Taenia saginata (8.20\%), Hymenolepis diminuta (2.19\%), Schiotosoma mansoni (0.27\%), Heterophyes hetrophyses (0.55\%) and Enterobius vermicularis $(0.27 \%)$. In N'Djamena the parasitism of those investigated was mainly (45.63\%) monospecific and poly-specific (bi-and tri-specific) in $15.03 \%$ of the causes while $39.34 \%$ of persons examined were free from all forms of protozoa and helminths. The epidemiology of pathogenic forms was linked to a lack of hygiene especially ignorance of the risk of faecal peril. It is therefore important to strengthen the health education of the population in this city in particular and throughout the country in general.

Keywords: faecal peril, protozoa, helminths, N'Djamena, Chad

\section{Introduction}

Intestinal parasites remain in many tropical countries a major public health problem. Their frequency continues to increase, and the number of asymptomatic carriers become increasingly important (Hamit et al., 2013). In 2016 WHO, estimated that more than 2 billion people around the world are infected with these parasites. Induced pathologies, considered neglected for a few decades along side major endemic diseases such as HIV/AIDS, tuberculosis, malaria, leprosy and atypical pneumonia (Dianou et al., 2004). represent one of the major causes of morbidity and mortality worldwide. They may not only cause immunodeficiency but also anemia especially in pregnant woman and children necessitating blood transfusion (OMS, 2010). By their spoliative action, the pathogens incriminated can significantly influence the nutritional status and the development of psychomotor of a person (Ouermi et al.,2012). In Chad, some works carried out in certain regions have shown the endemic character of gastrointestinal parasitosis. For example, Kostoingue et al.(2002) found the general rate of carriers to be 57.7\%: during the same decade, Hamit et al. (2008) reported on the city of N'Djamena the rate of global infestation of the pathogenic agents to $51 \%$. For a better fight against intestinal parasitosis, it is important to know their time of prevalence rates of intestinal parasites and to identify the risk of their transmission in the population of the city of N'Djamena. 


\section{Material and Methods}

\subsection{Study Zone}

This study was done in the city of N'Djamena: $14^{\circ} 58^{\prime} 0^{\prime \prime}$ and $15^{\circ} 9^{\prime} 0^{\prime \prime}$ East of longitude and $12^{\circ} 1^{\prime} 0$ " and $12^{\circ} 13^{\prime} 0^{\prime \prime}$ North of the latitude, around $280 \mathrm{~m}$ of altitude (Demba et al., 2018). N'Djamena, capital city of Chad (Central Africa) is constructed in a swampy site on the right of the Chari river, South of lake Chad and covers a space of 41.000 ha (Demba et al., 2018). It has a sahelian climate, characterised by two seasons: one long dry season from October to May and a rainy season from June to September with 200 to $500 \mathrm{~mm}$ of precipitation (Ministère de 1'Environnement de 1'Eau et de la Pêche, 2017). The annual thermal amplitudes are important with minimum temperatures between 14 to $23^{\circ} \mathrm{C}$ and the maximum temperatures between 33 to $42^{\circ} \mathrm{C}$ (DREM, 2013). The population of N'Djamena was evaluated at 600.000 in 1993 and reached 1.500.000 inhabitants in 2013 (INSEED, 2012) regrouped in 10 districts, a population growth of 3.61\% (INSEED, 2012).

\subsection{Study Population}

This transversal study was done between 24 October 2019 to 16 December 2019 and implicated 366 individuals as samples who were inhabitants of different quarters of N'Djamena. Persons included were between the ages of 1 to 77 years. Those excluded were subjects who were not living continuously for more than three months in N'Djamena during our research. And finally, those persons who refused to participate were also excluded. The

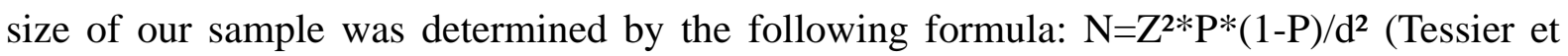
al.,2017), with $\mathrm{N}=$ size of the sample; $\mathrm{Z}$, coefficient of confidence $=1.96$; d, degree of precision $=0.05 ; \mathrm{p}=$ past prevalence, here that of Hamit et al., (2008) which was $51 \%$.

\subsection{Epidemiological Investigation}

Having the accord of the administrative authorities (Faculty of Human Health Sciences: $\mathrm{N}^{0}$ 28/PR/PM/MESRS/SG/U-NDJ/FSSH/2018 and Municipality of N'Djamena city: $\mathrm{N}^{0}$ 20/M/SG/DRH/SGCF/2018), the investigation respected all ethical rules linked to research on human beings (Tessier et al.,2017). All precautions were taken to preserve the confidentiality of data collected from the participants. Sampling in the population was done randomly by survey in clusters with three degrees. As first step, 20 clusters or primary sampling units (PSU) representing the quarters, where the study was randomly selected. Then 161 compounds or houses were randomly drawn from each PSU retained and then forming the units of the secondary sampling (USS). From each compound, a maximum of 3 individuals were randomly chosen. This was the third level of survey. Sociodemographic investigation was done the day before stool sampling. The participants were informed of the nature, the importance of the study and were also sensitized in order to get massive support for our investigation. After explanation of the objectives of our study and acquisition of the signatures and free consent from the subjects or their parents for children, an elaborated questionnaire on a survey sheet permitted us to obtain information on each participant and to know their general hygienic problems. At the end of this interrogation, a sterilized labelled, hermetically closed sampling jar containing $2 \mathrm{ml}$ of 10\% formalin (Odongo-Aginya, et al., 


\section{Macrothink}

2007; OMS, 2010) was handed to each participant to collect the stool sample in the morning between 8 and 9 AM. The jars and the corresponding survey sheets were collected and taken to the laboratory of the Faculty of Human Health Sciences of the University of N'Djamena for subsequent analysis.

\subsection{Laboratory Analysis}

Each sample was first examined macroscopically in order to appreciate the consistence and the colour of the stool, the presence of glair, adult worms, mucus and blood. Then microscopic examination was realized, including the direct technic, there after two complementary technics (formalin-ether enrichment for the detection of helminths eggs and the cysts of protozoa, and those quantitavely, for Kato Katz, for the detection of helminth eggs) (Odongo-Aginya, et al., 2007).

A subject was considered parasitized when the stool examined revealed the presence of a minimum of an intestinal parasite or one form of resistance (eggs or cysts).

\subsection{Biological Data Analysis}

The statistical analysis of the results was done by using SPSS Inc Version 18.0.2 the rates of carriage were compared using the test of Chi-2 $\left(\chi^{2}\right)$ with statistical significance $(\mathrm{p})$ value of < 0.05 (Bernard et Lapointe, 1987; Rambaud, 1997).

\section{Results}

A total of 366 individuals (171 females and 195 males) were examined during this study. Their ages vary from 1 to 77 years with an average of $27.2 \pm 18$ years and a median of 25 years. The copro-parasitological examination revealed a rate of infestation by at least one species of parasite in $60.66 \%$ (222/366 individuals) with $15.03 \%$ (55/366 individuals) of poly-parasitism and $45.63 \%$ (167/366 individuals) of mono-parasitism (table 1); $39.34 \%$ (144/366 individuals) subjects who were free from all forms of parasites. 


\section{Macrothink}

Table 1. Data on carriers of gastro-intestinal parasites

\begin{tabular}{|c|c|c|c|c|c|c|}
\hline $\begin{array}{c}\text { Type of } \\
\text { Parasitism }\end{array}$ & $\begin{array}{c}\text { Species } \\
\text { of parasites }\end{array}$ & Parasitized subjects & $(\%)$ & $\begin{array}{l}\text { Positive } \\
\text { cases }\end{array}$ & $(\%)$ & \\
\hline \multirow{9}{*}{$\begin{array}{l}\text { Mono- } \\
\text { specific }\end{array}$} & $\begin{array}{c}\text { E. } \\
\text { histolytical } \\
\text { dispar }\end{array}$ & 86 & $(23.5)$ & \multirow{9}{*}{167} & \multirow{9}{*}{\multicolumn{2}{|c|}{45.63}} \\
\hline & E. coli & 02 & $(0.55)$ & & & \\
\hline & Giardia intestinalis & 13 & $(3.55)$ & & & \\
\hline & A. lumbricoides & 17 & $(4.55)$ & & & \\
\hline & $\begin{array}{c}\text { En. } \\
\text { vermicularis }\end{array}$ & 01 & $(0.27)$ & & & \\
\hline & $\begin{array}{c}H . \\
\text { nana }\end{array}$ & 29 & (7.92) & & & \\
\hline & H. diminuta & 04 & $(1.09)$ & & & \\
\hline & S. mansoni & 01 & $(0.27)$ & & & \\
\hline & T. saginata & 14 & $(3.83)$ & & & \\
\hline \multirow{4}{*}{$\begin{array}{c}\text { Bi- } \\
\text { specific }\end{array}$} & $\begin{array}{l}\text { E. } \\
\text { histolityca/dispar }+H \text {. } \\
\text { nana }\end{array}$ & 20 & $(5.46)$ & \multirow[t]{4}{*}{45} & \multirow[t]{4}{*}{12.3} & \\
\hline & H. nana + T. saginata & 12 & $(3.28)$ & & & \\
\hline & $\begin{array}{l}\text { E. histolytica/dispar } \\
+ \text { A. lumbricoides }\end{array}$ & 11 & (3.01) & & & 15.03 \\
\hline & $\begin{array}{l}\text { He. heterophyes }+H . \\
\text { nana }\end{array}$ & 02 & $(0.55)$ & & & \\
\hline \multirow{3}{*}{$\begin{array}{l}\text { Tri- } \\
\text { specific }\end{array}$} & $\begin{array}{l}\text { E. histolytica/dispar } \\
+ \text { H. nana }+H . \\
\text { diminuta }\end{array}$ & 04 & $(1.09)$ & \multirow[t]{3}{*}{10} & \multirow[t]{3}{*}{2.73} & \\
\hline & $\begin{array}{l}\text { E. histolytica/dispar } \\
+ \text { A. lumbricoides }+ \\
\text { H. nana }\end{array}$ & 02 & $(0.55)$ & & & \\
\hline & $\begin{array}{l}\text { T. saginata }+\quad E . \\
\text { histolytica/dispar } \quad+ \\
\text { A. lumbricoides }\end{array}$ & 04 & $(1.09)$ & & & \\
\hline \multirow[t]{2}{*}{ Total } & \multicolumn{3}{|l|}{ Infected subjects } & 222 & \multirow{2}{*}{\multicolumn{2}{|c|}{$\frac{60.65}{39.35}$}} \\
\hline & Uninfected subjects & & & 144 & & \\
\hline
\end{tabular}

Legend: E= Entamoeba; En=Enterobius; H= Hymenolepis; He= heterophyes; $A=$ Ascaris; $T=$ Taenia, $S=$ Schistosoma

Seven species of helminths and three species of protozoa were identified (table 1 and 2) among the protozoa, Entamoeba histolytica/dispar 34.70\% was largely prevalent, followed by Giardia intestinalis (only $3.55 \%$ ); for helminths, infestation by Hymenolopis nana (18.95\%) was more frequent, followed by that of Ascaris lumbricoides $(9.25 \%)$ and Taenia saginata $(8.20 \%)$. 
Table 2. Prevalence of parasites

\begin{tabular}{c|c|c|c}
\hline \multicolumn{2}{c|}{ Species of parasites } & $\begin{array}{c}\text { Number of infected } \\
\text { subjects }\end{array}$ & $\begin{array}{c}\text { Rates of } \\
\text { Prevalence }(\%)\end{array}$ \\
\hline \multirow{4}{*}{ Protozoa } & Entamoeba histolytica/ dispar & 127 & $(34.70)$ \\
\cline { 2 - 4 } & Giardia intestinalis & 13 & $(3.55)$ \\
\cline { 2 - 4 } & Entamoeba coli & 02 & $(0.55)$ \\
\hline \multirow{4}{*}{ Helminths } & Hymenolepis nana & 69 & $(9.25)$ \\
\cline { 2 - 4 } & Ascaris lumbricoides & 34 & $(8.20)$ \\
\cline { 2 - 4 } & Taenia saginata & 30 & $(0.55)$ \\
\cline { 2 - 4 } & Hymenolepis diminuta & 08 & $(0.27)$ \\
\cline { 2 - 4 } & Heterophyes heterophyes & 02 & $(0.27)$ \\
\cline { 2 - 4 } & Enterobius vermicularis & 01 & 01 \\
\cline { 2 - 4 } & Schistosoma mansoni & & \\
\hline
\end{tabular}

The cysts of Giardia intestinalis were not diagnosed in the stools of inhabitants of the second; third, and tenth districts. The eggs of Entamoeba coli were found in one subject from the first district and another subject from the fourth district, the eggs of Ascaris lumbricoides were not found from participants of second, third, and tenth districts, same as the embryophores of Taenia saginata and the stools of subjects from the second, fourth and tenth districts. Infections with Enterobius vermicularis, Heterophyes heterophyes and Shistosoma mansoni were identified in the fifth district, finally Hymenolepis diminuta was found in the participants from first, fourth and tenth districts. In general, the carriage rates of pathogens were different $(\mathrm{P}<0.05)$ between the different administrative districts (table 3 ).

Table 3. Parasitism by different species at the level of the districts

\begin{tabular}{|c|c|c|c|c|c|c|c|c|c|c|c|}
\hline \multirow{3}{*}{ Parasite } & \multicolumn{11}{|c|}{ Districs } \\
\hline & $\begin{array}{l}\text { Ist } \\
(\mathrm{Ni}= \\
25)\end{array}$ & $\begin{array}{l}2 \mathrm{nd} \\
(\mathrm{Ni}= \\
9)\end{array}$ & $\begin{array}{l}\text { rd } \\
(\mathrm{Ni}= \\
39)\end{array}$ & $\begin{array}{l}4 \text { th } \\
(\mathrm{Ni}=14)\end{array}$ & $\begin{array}{l}5 \text { th } \\
(\mathrm{Ni}= \\
45)\end{array}$ & $\begin{array}{l}\text { 6th } \\
(\mathrm{Ni}= \\
54)\end{array}$ & $\begin{array}{l}7 \mathrm{th} \\
(\mathrm{Ni}= \\
67)\end{array}$ & $\begin{array}{l}\text { 8th } \\
(\mathrm{Ni}= \\
42)\end{array}$ & $\begin{array}{l}9 \text { th } \\
(\mathrm{Ni}= \\
48)\end{array}$ & $\begin{array}{l}10 \text { th } \\
(\mathrm{N}= \\
23)\end{array}$ & \multirow[t]{2}{*}{$\mathrm{P}$} \\
\hline & $\begin{array}{l}\mathrm{ni} \\
(\%)\end{array}$ & $\begin{array}{l}\text { ni } \\
(\%)\end{array}$ & $\begin{array}{l}\mathrm{ni} \\
(\%)\end{array}$ & $\begin{array}{l}\text { ni } \\
(\%)\end{array}$ & $\begin{array}{l}\mathrm{ni} \\
(\%)\end{array}$ & $\begin{array}{l}\text { ni } \\
(\%)\end{array}$ & $\begin{array}{l}\mathrm{ni} \\
(\%)\end{array}$ & $\begin{array}{l}\text { ni } \\
(\%)\end{array}$ & $\begin{array}{l}\text { ni } \\
(\%)\end{array}$ & $\begin{array}{l}\text { ni } \\
(\%)\end{array}$ & \\
\hline $\begin{array}{l}\text { E. his/ } \\
\text { dispar }\end{array}$ & \begin{tabular}{|l}
8 \\
$(32.0)$ \\
\end{tabular} & $\begin{array}{l}1 \\
(11.1) \\
\end{array}$ & \begin{tabular}{|l|}
8 \\
$(20.5)$ \\
\end{tabular} & \begin{tabular}{|l}
5 \\
$(35.7)$ \\
\end{tabular} & \begin{tabular}{|l|}
11 \\
$(24.4)$ \\
\end{tabular} & $\begin{array}{l}26 \\
(48.2)\end{array}$ & \begin{tabular}{|l|}
27 \\
$(40.3)$ \\
\end{tabular} & \begin{tabular}{|l|}
13 \\
$(31.0)$
\end{tabular} & $\begin{array}{l}23 \\
(47.9)\end{array}$ & \begin{tabular}{|l|}
5 \\
$(39.4)$ \\
\end{tabular} & 0.02 \\
\hline G. intest & $\begin{array}{l}1 \\
(4.0) \\
\end{array}$ & $\begin{array}{l}0 \\
(0.0) \\
\end{array}$ & $\begin{array}{l}0 \\
(0.0) \\
\end{array}$ & $\begin{array}{l}2 \\
(14.3) \\
\end{array}$ & $\begin{array}{l}0 \\
(0.0) \\
\end{array}$ & $\begin{array}{l}2 \\
(3.7) \\
\end{array}$ & $\begin{array}{l}3 \\
(1.5) \\
\end{array}$ & \begin{tabular}{|l|}
5 \\
$(11.9)$ \\
\end{tabular} & $\begin{array}{l}0 \\
(0.0)\end{array}$ & $\begin{array}{l}0 \\
(0.0) \\
\end{array}$ & 0.03 \\
\hline E. coli & $\begin{array}{l}1 \\
(4.0)\end{array}$ & $\begin{array}{l}0 \\
(0.0)\end{array}$ & $\begin{array}{l}0 \\
(0.0)\end{array}$ & $\begin{array}{l}1 \\
(7.2)\end{array}$ & $\begin{array}{l}0 \\
(0.0)\end{array}$ & $\begin{array}{l}0 \\
(0.0)\end{array}$ & $\begin{array}{l}0 \\
(0.0)\end{array}$ & $\begin{array}{l}0 \\
(0.0)\end{array}$ & $\begin{array}{l}0 \\
(0.0)\end{array}$ & $\begin{array}{l}0 \\
(0.0)\end{array}$ & \\
\hline A. lumbricoides & \begin{tabular}{|l|} 
\\
$(8.0)$ \\
\end{tabular} & $\begin{array}{l}0 \\
(0.0)\end{array}$ & $\begin{array}{l}0 \\
(0.0)\end{array}$ & \begin{tabular}{|l|}
3 \\
$(21.4)$ \\
\end{tabular} & \begin{tabular}{|l|}
11 \\
$(24.4)$ \\
\end{tabular} & \begin{tabular}{l|}
8 \\
$(14.8)$ \\
\end{tabular} & $\begin{array}{l} \\
(3.0) \\
\end{array}$ & $\begin{array}{l}4 \\
(9.5)\end{array}$ & $\begin{array}{l}4 \\
(8.3)\end{array}$ & $\begin{array}{l}0 \\
(0.0)\end{array}$ & 0.01 \\
\hline Hy. nana & \begin{tabular}{|l|}
4 \\
$(16.0)$ \\
\end{tabular} & $\begin{array}{l}1 \\
(11.1) \\
\end{array}$ & \begin{tabular}{|l|}
6 \\
$(15.4)$ \\
\end{tabular} & $\begin{array}{l}0 \\
(0.0) \\
\end{array}$ & \begin{tabular}{|l|}
6 \\
$(13.3)$ \\
\end{tabular} & $\begin{array}{l}13 \\
(24.1) \\
\end{array}$ & \begin{tabular}{|l|}
9 \\
$(13.4)$ \\
\end{tabular} & \begin{tabular}{|l|}
13 \\
$(31.0)$ \\
\end{tabular} & $\begin{array}{l}11 \\
(22.9) \\
\end{array}$ & $\begin{array}{l}6 \\
(8.7) \\
\end{array}$ & 0.3 \\
\hline T. saginata & \begin{tabular}{|l|}
2 \\
$(8.0)$ \\
\end{tabular} & $\begin{array}{l}0 \\
(0.0) \\
\end{array}$ & \begin{tabular}{|l|}
10 \\
$(25.6)$ \\
\end{tabular} & \begin{tabular}{|l|}
0 \\
$(0.0)$ \\
\end{tabular} & \begin{tabular}{|l|}
6 \\
$(13.3)$ \\
\end{tabular} & $\begin{array}{l}3 \\
(5.6) \\
\end{array}$ & \begin{tabular}{|l|}
3 \\
$(4.5)$ \\
\end{tabular} & \begin{tabular}{|l|}
1 \\
$(2.4)$ \\
\end{tabular} & $\begin{array}{l}5 \\
(10) \\
\end{array}$ & \begin{tabular}{|l|}
0 \\
$(0.0)$ \\
\end{tabular} & 0.01 \\
\hline $\begin{array}{l}\text { En. } \\
\text { vermicularis }\end{array}$ & $\begin{array}{l}0 \\
(0.0) \\
\end{array}$ & $\begin{array}{l}0 \\
(0.0) \\
\end{array}$ & $\begin{array}{l}0 \\
(0.0) \\
\end{array}$ & \begin{tabular}{|l|}
0 \\
$(0.0)$ \\
\end{tabular} & $\begin{array}{l}1 \\
(2.2) \\
\end{array}$ & $\begin{array}{l}0 \\
(0.0)\end{array}$ & \begin{tabular}{|l|}
0 \\
$(0.0)$ \\
\end{tabular} & $\begin{array}{l}0 \\
(0.0) \\
\end{array}$ & $\begin{array}{l}0 \\
(0.0)\end{array}$ & $\begin{array}{l}0 \\
(0.0) \\
\end{array}$ & 0.01 \\
\hline H. heterophyes & \begin{tabular}{|l|}
0 \\
$(0.0)$ \\
\end{tabular} & $\begin{array}{l}0 \\
(0.0) \\
\end{array}$ & $\begin{array}{l}0 \\
(0.0) \\
\end{array}$ & \begin{tabular}{|l|}
0 \\
$(0.0)$ \\
\end{tabular} & \begin{tabular}{|l} 
\\
$(4.4)$ \\
\end{tabular} & $\begin{array}{l}0 \\
(0.0)\end{array}$ & \begin{tabular}{|l|}
0 \\
$(0.0)$ \\
\end{tabular} & \begin{tabular}{|l|}
0 \\
$(0.0)$ \\
\end{tabular} & $\begin{array}{l}0 \\
(0.0)\end{array}$ & \begin{tabular}{|l|}
0 \\
$(0.0)$ \\
\end{tabular} & 0.01 \\
\hline Hy. diminuta & \begin{tabular}{|l|} 
\\
$(8.0)$ \\
\end{tabular} & $\begin{array}{l}0 \\
(0.0)\end{array}$ & $\begin{array}{l}0 \\
(0.0)\end{array}$ & \begin{tabular}{|l|}
3 \\
$(21.4)$ \\
\end{tabular} & $0(0.0)$ & $\begin{array}{l}1 \\
(1.9)\end{array}$ & \begin{tabular}{|l|}
0 \\
$(0.0)$ \\
\end{tabular} & $\begin{array}{l}0 \\
(0.0) \\
\end{array}$ & $\begin{array}{l}0 \\
(0.0)\end{array}$ & \begin{tabular}{|l}
2 \\
$(8.7)$ \\
\end{tabular} & 0.01 \\
\hline S. mansoni & $\begin{array}{l}0 \\
(0.0)\end{array}$ & $\begin{array}{l}0 \\
(0.0)\end{array}$ & $\begin{array}{l}0 \\
(0.0)\end{array}$ & $\begin{array}{l}1 \\
(7.1)\end{array}$ & $\begin{array}{l}0 \\
(0.0)\end{array}$ & $\begin{array}{l}0 \\
(0.0)\end{array}$ & $\begin{array}{l}0 \\
(0.0)\end{array}$ & $\begin{array}{l}0 \\
(0.0)\end{array}$ & $\begin{array}{l}0 \\
(0.0) \\
\end{array}$ & $\begin{array}{l}0 \\
(0.0)\end{array}$ & 0.01 \\
\hline
\end{tabular}


Legend: $\mathrm{N}_{\mathrm{i}}=$ sample by district; $\mathrm{n}_{\mathrm{i}}=$ number of infested persons; $(\%)=($ percentage $) ; E=$ Entamoeba ;

his=histolytica,intestinalisG=Giardia $; A=$ Ascaris $;$ Hy.=Hymenolopis $;$ T=Taenia $;$ En=Entero bius $;$ H=Heterophys $;$ S=Schistosoma $; \mathrm{P}=\mathrm{P}$ value

This overall index dropped $(\mathrm{P}=0.01)$ inversely to the monthly income of the subject (table 4$)$. Suburban inhabitants are more frequently infected than those of urban centre $(\mathrm{P}=0.01)$, while habitat modernization has reduced $(\mathrm{P}=0.01)$; these rates of carriers. Despite the non-significant nature of the test $(\mathrm{P}=0.29)$, the percentage of the infection seemed to increase with the size of the family (table 4$)$.

Table 4. Relations between sociodemographic data and global parasitism in N'Djamena

\begin{tabular}{|c|c|c|c|c|c|}
\hline \multicolumn{2}{|c|}{ Characteristics } & $\begin{array}{l}\text { Number of } \\
\text { subjects } \\
\text { examined }\end{array}$ & $\begin{array}{lr}\text { Number } & \text { of } \\
\text { Positive } & \text { subjects } \\
(\%) & \\
\end{array}$ & $\begin{array}{l}\text { Number } \quad \text { of } \\
\text { Negative subjects } \\
(\%)\end{array}$ & $\mathrm{P}$ value \\
\hline \multirow[t]{2}{*}{ Gender } & Male & 195 & $120(61.54)$ & $75(38.46)$ & \multirow[t]{2}{*}{0.7} \\
\hline & Femele & 171 & $102(59.65)$ & $69(40.35)$ & \\
\hline \multirow[t]{10}{*}{ Districs } & $1^{\text {st }}$ & 25 & $16(64)$ & $9(36.0)$ & \multirow[t]{10}{*}{0.01} \\
\hline & $2^{\text {nd }}$ & 09 & $01(11.11)$ & $4(44.45)$ & \\
\hline & $3^{\text {rd }}$ & 39 & $19(48.72)$ & $20(51.28)$ & \\
\hline & $4^{\text {th }}$ & 14 & $10(71.4)$ & $8(57.15)$ & \\
\hline & $5^{\text {th }}$ & 45 & $30(66.67)$ & $15(33.33)$ & \\
\hline & $6^{\text {th }}$ & 54 & $37(68.52)$ & $17(31.48)$ & \\
\hline & $7^{\text {th }}$ & 67 & $34(50.75)$ & $33(49.25)$ & \\
\hline & $8^{\text {th }}$ & 42 & $29(69.05)$ & $13(30.95)$ & \\
\hline & $9^{\text {th }}$ & 48 & $36(75)$ & $12(25)$ & \\
\hline & $10^{\text {th }}$ & 23 & $10(43.48)$ & $13(56.52)$ & \\
\hline \multirow{5}{*}{$\begin{array}{l}\text { Monthly } \\
\text { (FCFA) }\end{array}$} & $00-75000$ & 37 & $28(75.68)$ & $9(24.32)$ & \multirow[t]{5}{*}{0.01} \\
\hline & $76000-150000$ & 29 & $26(89.66)$ & $3(10.34)$ & \\
\hline & $151000-250000$ & 55 & $33(60)$ & $22(40)$ & \\
\hline & $251000-400000$ & 67 & $39(58.21)$ & $28(41.79)$ & \\
\hline & $>400000$ & 178 & $96(53.93)$ & $82(46.07)$ & \\
\hline \multirow[t]{2}{*}{ Origin } & Urban center & 309 & $177(57.28)$ & $132(42.72)$ & \multirow[t]{2}{*}{0.01} \\
\hline & Peri-urban & 57 & $45(78.95)$ & $12(21.05)$ & \\
\hline \multirow[t]{3}{*}{ Type of residence } & Modern & 60 & $26(43.33)$ & $34(56.67)$ & \multirow[t]{3}{*}{0.01} \\
\hline & Semi-modern & 213 & $128(60.09)$ & $85(39.91)$ & \\
\hline & Mud house & 93 & $68(73.12)$ & $25(26.88)$ & \\
\hline \multirow[t]{4}{*}{ Persons/compound } & $<5$ & 43 & $25(58.14)$ & $18(41.86)$ & \multirow[t]{4}{*}{0.29} \\
\hline & $6-10$ & 123 & $68(55.28)$ & $55(44.72)$ & \\
\hline & $11-20$ & 101 & $62(61.39)$ & $39(38.61)$ & \\
\hline & $\geq$ à 20 & 99 & $67(67.68)$ & $32(32.32)$ & \\
\hline
\end{tabular}

The amelioration or modernisation of water supply process also showed an inclination of reducing the frequency of gastro-intestinal parasitosis $(\mathrm{P}=0.05)$ (table 5). This reduction was noticed with subjects washing their hands with soap before meal (table 5). Meals eaten collectively seemed to favour the transmission of parasites. The modernizations of toilets reduce the parasite transmission, same as washing of anal regions with soap after stool (table 5). The rate of carriers of parasites looked relatively much higher or $63.98 \%$ and $65.52 \%$, but not significant $(\mathrm{p}=0.24$ and 0.12 ) in persons cohabiting respectively with pets or domestic animals (table 5). 
Table 5. Relationship between behavioral data and global parasitism in N'Djamena

\begin{tabular}{|c|c|c|c|c|c|}
\hline \multicolumn{2}{|c|}{ Caracterisitics } & \multirow{3}{*}{$\begin{array}{l}\text { Number of examined subjects } \\
161 \\
189\end{array}$} & \multirow{2}{*}{$\begin{array}{l}\begin{array}{l}\text { Number of } \\
\text { positive } \\
\text { subjects }(\%)\end{array} \\
91(56,52)\end{array}$} & \multirow{2}{*}{$\begin{array}{l}\begin{array}{l}\text { Number of } \\
\text { negative } \\
\text { subjects }(\%)\end{array} \\
70(43,48)\end{array}$} & \multirow{2}{*}{$\begin{array}{l}\text { P value } \\
0,05\end{array}$} \\
\hline \multirow[b]{3}{*}{ Drinking water } & SNE & & & & \\
\hline & Driling water & & $117(61,90)$ & $72(38,10)$ & \\
\hline & Well water & 16 & $14(87,50)$ & $2(12,5)$ & \\
\hline \multirow{2}{*}{$\begin{array}{l}\text { Hand washing } \\
\text { before meals }\end{array}$} & With soap & 276 & $156(56,52)$ & $120(43,48)$ & \multirow[t]{2}{*}{0.01} \\
\hline & Without soap & 90 & $66(73,33)$ & $24(26,66)$ & \\
\hline \multirow[t]{2}{*}{ Feeding habits } & Collective & 347 & $214(61,67)$ & $133(38,33)$ & \multirow[t]{2}{*}{0.09} \\
\hline & Individual & 19 & $8(42,11)$ & $11(57,89)$ & \\
\hline \multirow[t]{3}{*}{ Type of toilet } & Modern & 67 & $29(43,28)$ & $38(56,72)$ & \multirow[t]{3}{*}{0.01} \\
\hline & Semi-modern & 169 & $105(62,13)$ & $64(37,87)$ & \\
\hline & Traditional & 130 & $88(67.69)$ & $42(32.31)$ & \\
\hline \multirow[t]{3}{*}{$\begin{array}{l}\text { Anal } \\
\text { cleaning }\end{array}$} & $\begin{array}{l}\text { Water without } \\
\text { soap }\end{array}$ & 82 & 61(74.39) & $21(25.61)$ & \multirow[t]{3}{*}{0.01} \\
\hline & Toilet paper & 207 & $124(59.90)$ & $83(40.10)$ & \\
\hline & $\begin{array}{l}\text { Water with } \\
\text { soap }\end{array}$ & 77 & $37(48.05)$ & $40(51.95)$ & \\
\hline \multirow{2}{*}{$\begin{array}{l}\text { Cohabitation } \\
\text { with pets } \\
\text { compagnies }\end{array}$} & Yes & 161 & $103(63.98)$ & $58(36.02)$ & \multirow[t]{2}{*}{0.24} \\
\hline & No & 205 & $119(58.05)$ & $86(41.95)$ & \\
\hline \multirow{2}{*}{$\begin{array}{l}\text { Cohabitation } \\
\text { with livestock }\end{array}$} & Yes & 145 & $95(65.52)$ & $50(34.48)$ & \multirow[t]{2}{*}{0.12} \\
\hline & No & 221 & $127(57.47)$ & $94(42.53)$ & \\
\hline
\end{tabular}

Legend: SNE= National Water supply company in Chad

Parasitism by gender (figure 1) was different $(\mathrm{P}<0.05)$ for Entamoeba histolytica/dispar was the highest proportion of parasites located in males; meanwhile H.nana and H.dimunita were higher in female. G.intestinalis, A. Lumbricoides, T.saginata were all higher in males; other parasites like E.coli, E.vermicularis and S.mansoni were only found in males. It is interesting to know that $H$. heterophyse has the same number in males as in females. Generally speaking as seen in the histogram below men are greater carriers of these parasites.

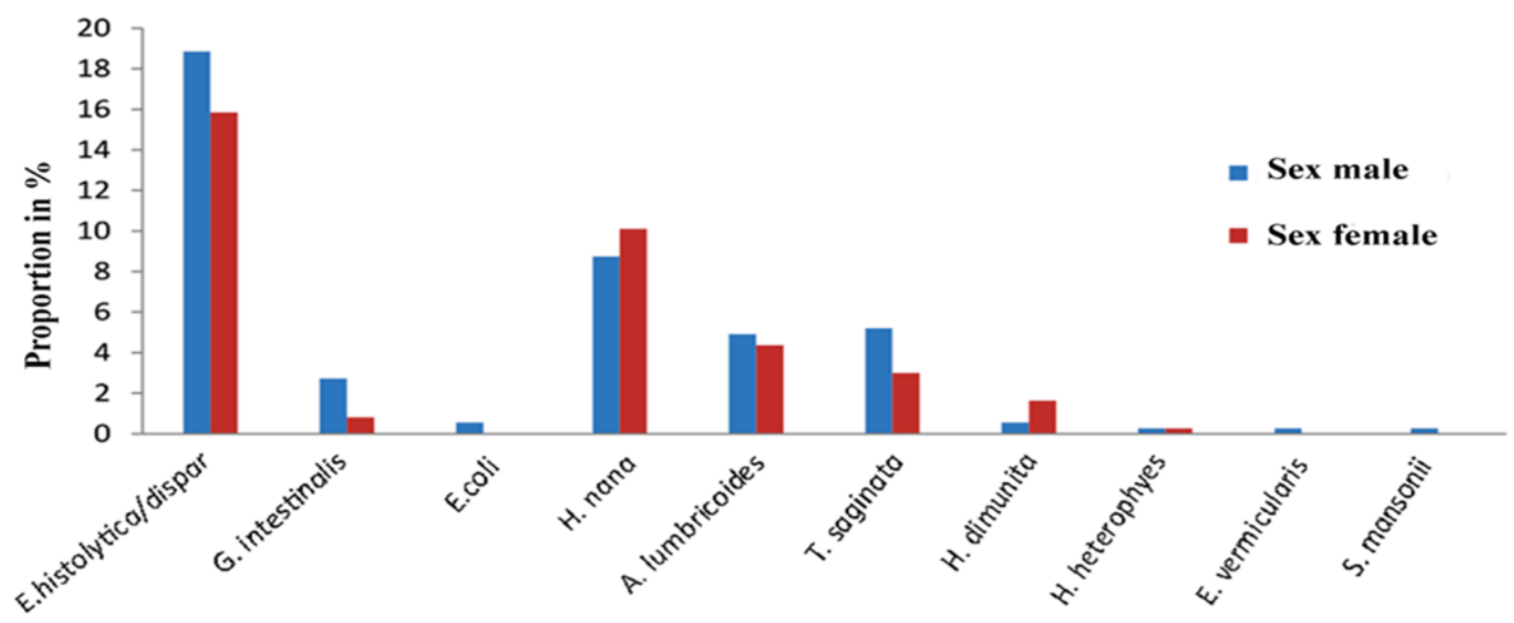

Parasites

Figure 1. Prevalence by gender 
Figure (2) below shows that the direct methods allowed us to diagnose 183 (82.4\%) stools containing at least one parasite. Complementary technics showed 222 positive stools, or either a difference of 39 (17.6\%) stools which were falsely declared negative by the direct observation methods.
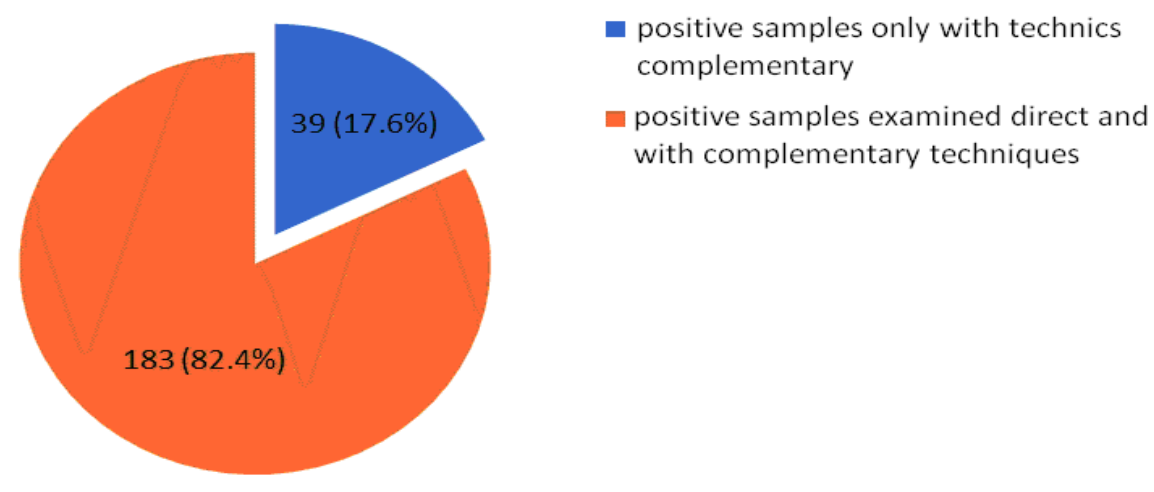

Figure 2. Detection rate of the parasitic elements by direct examination and with the complementary techniques

\section{Discussion}

This work was aimed at updating the data on the rate of gastrointestinal parasitosis among the population of the city of N'Djamena, following the work done previously (Hamit et al., 2008) and to determine the risk factors. It was found that $60.66 \%$ of the persons infected were carriers of at least one species of parasite, this value exceeds that obtained by above authors in 2008 , which was $51 \%$. This increase is due to the swampy zone of the city of N'Djamena, by the peripheric extension of quarters and because of more and more over population due to the rural exodus in progress since the last 10 years (Hamit et al., 2013). The populations of these quarters are also characterized by great poverty, illiteracy, a lack of respect for hygiene rules, a virtual absence of water supply and sanitation system. All these weaknesses are known as determinants of the spread of infection agents (Bundy et al., 2009). Among the listed species, E. histolytica/dispar and Hymenolepis nana, are known for their presence in unhealthy environment and lack of hygiene (Hamit et al., 2008) were more diagnosed. Mono-parasitism concerned $45.63 \%$ of our samples, bi-parasitism and tri-parasitism infected $12.3 \%$ and $2.73 \%$ of the subjects. This result still shows a very low level of sanitary hygiene, food and faecal and the existence of precarious living conditions (El Guamri et al., 2009) among the inhabitants of N'Djamena in general who do not seem to appropriate the risks of faecal peril. For the parasites more prevalent, no difference in infection was noticed between the genders with E. histolytica/dispar, G.intestinalis, T.saginata and A.lumbricoides, contrary to Hymenolopis nana $(\mathrm{P}<0.05)$ that males were the most parasitized. This last situation may be partially explained by the eating habits of men who sometimes eat out of their houses: uncooked foods, cake, bread and other products that may be contaminated. Flour products containing fleas or mealworms carrying cysticercoids and known as intermediate hosts in the transmission of hymenolepiosis (Mohammad and Hegazi, 2007). Concerning 
sociodemographic factors, a significant association $(\mathrm{P}<0.05)$ was found between parasitism and the living environment [district], the monthly revenue of the subject and the type of home. Parasitism $(60,66 \%)$ was particularly higher in very poor individuals who are living in the periphery of the city, staying in mud houses. These results are comparable with those obtained by Agbaya et al., 2004 in Agboville, South of Ivory Coast and North-East of Brazil (Perez et al., 2000).

A relationship has been highlighted between parasitism and the type of water consumed, the type of toilets used, and the mode to clean the anal areas after stool, and the methods of washing hands before meals. Indeed, the consumption of well water, the utilization of traditional toilets, cleaning of buttocks with non-soaped water after stool and washing of hands without soap before eating predispose the participants to contact gastrointestinal parasitosis (Agbaya et al., 2004). The proximity with pets or livestock did not really influence the rate of parasitic carriage as noted with children in Marrakech (Benzalim and Bouskraoui, 2010).

The utilization of complementary techniques (formalin-ether concentration and the Kato katz concentration technic) allowed us to improve the coprological diagnosis. This observation coincides with that of (Hamit et al., 2013) within two ecological zones of Chad. These authors found that the direct observation method was only $65.3 \%$ efficient as compound with Kato-Katz. Our study confirms that, complementary methods should be recommended for crowd research (Tchuenté et al., 2012). It is suggested that complimentary techniques should also be constantly applied in hospitals in Chad. These technics have the advantage of being relatively simple, fast, less expensive and consequently, perfectly adapted to developing countries.

\section{Conclusions}

Intestinal parasites are spread all over the world. Their prevalence is particularly higher in Tropical and Sahelian regions. In the city of N'Djamena, $60.66 \%$ of the participants in our study were carriers of a minimum of one parasitic species. This value is higher than previous studies. A strong prevalence of Entamoeba histolyticaldispar and Giardia intestinalis was noticed in the case of protozoa, as for helminths, Hymenolepis nana followed by Ascaris lumbricoides were frequently diagnosed. In order to reduce this prevalence, an amelioration of hygienic conditions, access to clean drinking water and a change of mentality or habits are necessary and urgent. An adequate strategy to prevent gastrointestinal parasitosis should be put in place. The consolidation of progress concerning collective hygiene and the amelioration of technics for stool examination for better medical prescriptions are necessary in order to achieve better fight against gastrointestinal disease.

\section{References}

Agbaya, S. S. O., Yavo, W., Menan, E. I. H., Kouadio, L. P., \& Koné, M. (2004). Helminthiases intestinales chez les enfants d'âge scolaire: résultats préliminaires d'une étude prospective à Agboville dans le sud de la Côte d'Ivoire. Cahiers d'études et de recherches francophones/Santé, 14(3), 143-147. 


\section{Al Macrothink}

Journal of Biology and Life Science

ISSN 2157-6076

2020, Vol. 11, No. 2

Benzalim, M., \& BOUSKRAOUI, M. (2010). Dépistage des parasites intestinaux chez les enfants consultant a l'hôpital de jour de pédiatrie au chu $m$ vi a Marrakech (Doctoral dissertation, thèse doctorat en médecine Universite Cadi Ayyad, Faculté de Médecine et de Pharmacie Marrakech).

Bernard, P. M., \& Lapointe, C. (1987). Mesures statistiques en épidémiologie. PUQ.

Bundy, D. A., Kremer, M., Bleakley, H., Jukes, M. C., \& Miguel, E. (2009). Deworming and development: asking the right questions, asking the questions right. PLoS Negl Trop Dis, 3(1), e362. https://doi.org/10.1371/journal.pntd.0000362

Demba Kodindo, K., Kana-Mbang, A., Moundai, T., Nakebang Fadel, A., Yangalbé-Kalnoné, E., Mahamat Oumar, A., ... Kerah-Hinzoumbé, C. (2018). Sensibilité de Anopheles gambiae sl et Culex quinquefasciatus à divers insecticides en milieu urbain à N'Djamena, Tchad. Med Sante Trop, 154-157. https://doi.org/10.1684/mst.2018.0778

Dianou, D., Poda, J. N., Savadogo, L. G., Sorgho, H., Wango, S. P., \& Sondo, B. (2004). Parasitoses intestinales dans la zone du complexe hydroagricole du Sourou au Burkina Faso. VertigO-la revue électronique en sciences de l'environnement, 5(2). https://doi.org/10.4000/vertigo.3369

DREM (2013) Ministère de l'Environnement. Division de climatologie. Rapport de février 2013 de la Direction des ressources en eaux et de la météorologie du Tchad, 23p.

El Guamri, Y., Belghyti, D., Achicha, A., Tiabi, M., Aujjar, N., Barkia, A., ... Bouachra, H. (2009, March). Enquête épidémiologique rétrospective sur les parasitoses intestinales au Centre hospitalier provincial El Idrissi (Kénitra, Maroc): bilan de 10 ans (1996-2005). In Annales de Biologie Clinique (Vol. 67, No. 2, pp. 191-202). https://doi.org/10.1684/abc.2009.0313

Hamit M A., Tidjani, A., Otchom, B. B., Tidjani, M. T., \& Felix, B. B. C. (2013). An epidemiological assessment of the infectious forms of intestinal helminths in school children from Chad. Journal of Biology and Life Sciences, 4(2), 341. https://doi.org/10.5296/jbls.v4i2.4258

Hamit, M. A., Tidjani, M. T., \& Bilong, C. B. (2008). Recent data on the prevalence of intestinal parasites in N'Djamena, Chad Republic. African Journal of Environmental Science and Technology, 2(12), 407-411.

INSEED (2012) Ministère de la coopération du Tchad. Institut nationale de la statistique des études économiques et démographique/ Rapport du 2éme recensement général de la population et de l'habitat 2010, 235p.

Kostoingue, B., Tidjani, M. T., Mbaideji, F., \& Alio, H. M. (2002). Prevalence des parasitoses intestinales chez les enfants de 0 a 5 ans dans la ville de N'Djamena. Médecine d'Afrique noire, 49(12), 533-536. 
Ministère de l'Environnement de l'Eau et de la Pêche (République du Tchad), N'Djaména (2017) Stratégies de Lutte Contre les Changements Climatiques au Tchad (SNLCC), 2017. 55p

Mohammad, M. A., \& Hegazi, M. A. (2007). Intestinal permeability in Hymenolepis nana as reflected by non invasive lactulose/mannitol dual permeability test and its impaction on nutritional parameters of patients. Journal of the Egyptian Society of Parasitology, 37(3), 877.

Odongo-Aginya, E. I., Narcis, K., Ludwig, S., Wabinga, H., Fenwick, A., \& Montresor, A. (2007). Substitution of malachite green with nigrosin-eosin yellow stain in the Kato-Katz method: microscopical appearance of the helminth eggs. African health sciences, 7(1).

OMS (2010) Profil épidémiologique des maladies transmissibles. République Centrafricaine et Tchad. Relief-Web-report-http://reliefweb.int/node/351755, 275p

Ouermi, D., Karou, D. S., Ouattara, I., Gnoula, C., Pietra, V., Moret, R., ... Simpore, J. (2012). Prévalence des parasites intestinaux de 1991 à 2010 au centre médical Saint-Camille de Ouagadougou (Burkina Faso). Médecine et Santé Tropicales, 22(1), 40-44. https://doi.org/10.1684/mst.2012.0008

Perez, E., Gazin, P., Furtado, A., Miranda, P., Marques, N. M., Silva, M. R., \& Varela, R. (2000). Parasitoses intestinales et schistosomose en milieu urbain, en région littorale et en région semi-aride du Nord-Est du Brésil. Cahiers Santé, 10(2), 127-130.

Rambaud, D. (1997). L'échantillonnage: du prélèvement à l'analyse.

Tchuenté, L. A. T., Ngassam, R. I. K., Sumo, L., Ngassam, P., Noumedem, C. D., Nzu, D. D. O. L., ... Tarini, A. (2012). Mapping of schistosomiasis and soil-transmitted helminthiasis in the regions of centre, east and west Cameroon. PLoS Negl Trop Dis, 6(3), e1553. https://doi.org/10.1371/journal.pntd.0001553

Tessier, C., Viallet, F., Mary, A., Tondu, B., Baudouin, L., Ducournau, P., ... Dessens, N. (2017). Formation Doctorale" Éthique de la recherche et intégrité scientifique"

World Health Organization. (2006). Preventive chemotherapy in human helminthiasis. Coordinated use of anthelminthic drugs in control interventions: a manual for health professionals and programme managers. World Health Organization.

\section{Copyright Disclaimer}

Copyright for this article is retained by the author(s), with first publication rights granted to the journal.

This is an open-access article distributed under the terms and conditions of the Creative Commons Attribution license (http://creativecommons.org/licenses/by/4.0/). 